\title{
AUTOMATIC 3D MODELING DEVELOPMENT AND APPLICATION FOR HYDRAULIC CONSTRUCTION
}

\author{
Po-Han Chen \\ Assistant Professor \\ School of Civil and Environmental Engineering, \\ Nanyang Technological University, \\ Singapore 639798 \\ cphchen@ntu.edu.sg
}

\author{
Nguyen Thi Lan Truc \\ Master of Engineering Student \\ School of Civil and Environmental Engineering, \\ Nanyang Technological University, \\ Singapore 639798 \\ nguy0050@ntu.edu.sg
}

\begin{abstract}
Nowadays, the application of 3D models is increasing in almost every field, especially the AEC (Architecture, Engineering, and Construction) industry. Most 3D models are generated through human manipulation with the use of CAD software like 3D Max, AutoCAD or Maya. These software packages are very efficient in 3D modeling, but sometimes they are not easy to use and experience might be required. Moreover, traditional 3D modeling is time-consuming, as manual input is required for each component as well as for the whole scene. Therefore, it is difficult for a person to build 3D models without good 3D modeling skills and the knowledge of 3D modeling tools, although CAD software provides many facilitating functions.

In the hydraulic engineering domain, 3D models are used not only to illustrate the realistic view before construction, but also to measure the construction's volume in order to estimate cost based on cut-and-fill volume quantity, concrete volume quantity, etc. Therefore, 3D modeling is crucial and greatly needed in planning hydraulic construction. Since 3D modeling for hydraulic facilities requires accuracy and details, it would need a lot of time, effort and prior experience to manually build the whole model from scratch.

To meet this need, an approach of automatic 3D modeling is proposed in this paper. The main purpose is to help the user build a 3D model of hydraulic construction in less time and with less manual operation. Also, the proposed approach aims to provide easy-to-use features for those who do not have much experience in $3 \mathrm{D}$ modeling.

To demonstrate the proposed approach, an application is developed in the AutoCAD environment to automatically generate a 3D sluice model, a model of artificial passageway for water fitted with a valve or gate to stop or regulate water flow.

With this approach, users no longer have to manually build 3D models step by step. A 3D model could be automatically generated shortly after the input of data. As most steps are automatically done by the computer, the result would be of high accuracy. A complete 3D sluice model could be created from a sketch without much effort and time spent comparing to the traditional step-by-step manual input and operation.
\end{abstract}

\section{KEYWORDS}

Automatic, 3D modeling, VisualLISP, sluice 


\section{AIMS}

Nowadays, the application of 3D models is increasing in almost every field, especially the AEC (Architecture, Engineering, and Construction) industry. Most 3D models are generated through human manipulation with the use of CAD software like 3D Max, AutoCAD or Maya. These software packages are very efficient in 3D modeling, but sometimes they are not easy to use and experience might be required. Moreover, traditional 3D modeling is timeconsuming, as manual input is required for each component as well as for the whole scene. Therefore, it is difficult for a person to build 3D models without good 3D modeling skills and the knowledge of 3D modeling tools, although CAD software provides many facilitating functions. In the hydraulic engineering domain, 3D models are used not only to illustrate the realistic view before construction, but also to measure the construction's volume in order to estimate cost based on cut-and-fill volume quantity, concrete volume quantity, etc. Therefore, 3D modeling is crucial and greatly needed in planning hydraulic construction.

Since 3D modeling for hydraulic facilities requires accuracy and details, it would need a lot of time, effort and prior experience to manually build the whole model from scratch.

To meet this need, an approach of automatic 3D modeling is proposed in this paper. The main purpose is to help the user build a 3D model of hydraulic construction in less time and with less manual operation. Also, the proposed approach aims to provide easy-to-use features for those who do not have much experience in 3D modeling.

\section{SIGNIFICANCES}

The automatic 3D modeling approach could significantly reduce time, effort and experience required to create 3D models. With this approach, users no longer have to build 3D models step by step. A 3D model could be automatically generated shortly after the input of data.

As most steps are automatically done by the computer, the result would be of high accuracy. Hence, the application output could be used as product utili- zation such as rendering reality view and cost estimate through volume determination.

\section{METHODS}

The application is developed using VisualLISP, a tool for code creation in AutoCAD environment. It is a full-featured, interpretive programming language that can be used to call AutoCAD commands, system variables, and dialog boxes [1]. It can also create user-defined functions and macros in order to facilitate AutoCAD users and increase performance and productivity. The integrated development environment (IDE) offered by VisuaLISP reduces the required development time and makes it easier and faster for users and developers to create, debug and deliver AutoLISP-based applications.

Application analyzing and design steps are as follows:

1. To determine the application parameters.

2. To create application's GUI (Graphical User Interface).

3. To develop application functions and macros.

4. To test and verify the correctness and robustness of the application.

To demonstrate the proposed approach, an application is developed in the AutoCAD environment to automatically generate a 3D sluice model, a model of artificial passageway for water fitted with a valve or gate to stop or regulate water flow.

\subsection{Determining application parameters}

The application parameters (or input data) are the primary dimensions of the sluice, the type for each sluice component, and the position of the sluice model in the AutoCAD environment.

A sluice structure is usually constructed by several components and each component is designed according to its function (Figure 1-1). Hence, each component has its specific shape that is determined by its dimensions. However, some dimensions are crucial as they determine the component's shape. Therefore, all the crucial dimensions for each component should be identified and selected as its input parameters. The fewer the number of parameters, the more user-friendly the application. Other dimensions are extrapolated from the primary ones or from other 
sources according to the constraints of the criteria and the relations among components.

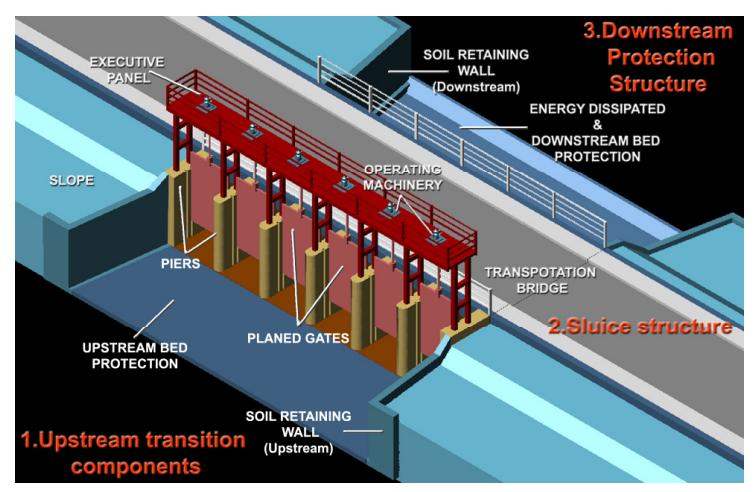

Figure 1-1. Sluice components

Options refer to the different types of components available for selection, such as sluice gates, piers, and so on. The options enable the users to model various types of sluices.

To locate the sluice model in AutoCAD, an insertion point is required. The insertion point is also needed to align and assemble all the components together to form a complete sluice model.

\subsection{Creation of application GUI}

Based on the number of input parameters corresponding to each component, an application GUI (Graphical User Interface) could be developed to allow people to interact with the application. The application GUI comprises widgets such as graphical icons, visual indicators along with text, labels or text navigation to present the information and actions available to users.

As VisualLISP does not support GUI's wizard (which leads users through a sequence of steps to build their own application interface), the Dialog Control Language (DCL) is introduced instead to perform this function. DCL, a built-in GUI mini language for creating simple graphical dialogs within AutoCAD, allows the user to interact with the AutoCAD environment through basic widgets called tiles, such as edit boxes, buttons, checkboxes, radio buttons and list boxes. In order to develop a dialog with DCL, all tiles involved in this dialog have to be constructed in a hierarchical tree in an inverted or- der. Specifically, a parent tile has to contain children tiles, successively. After that, DCL relies on the tree to develop the corresponding GUI. The hierarchical tree needs to build prior to GUI programming. Figure 1-2 is an example of developing a GUI for a component through hierarchical tree of tiles.

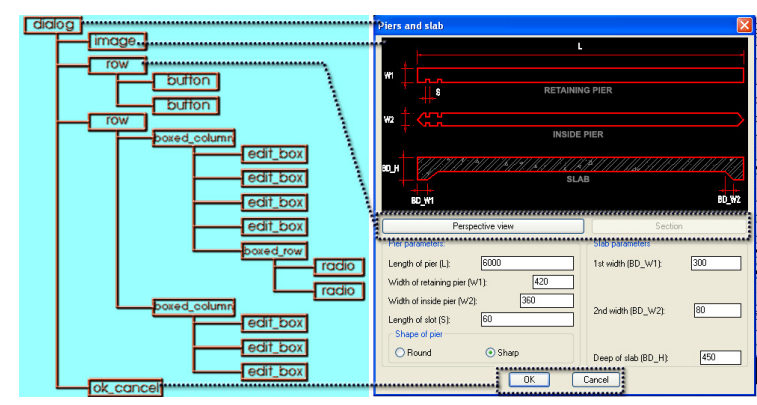

Figure 1-2. An example of application GUI and its hierarchical tree

After all needed GUIs are created with DCL, they are in the DCL format. Therefore, VisualLISP is used to make GUIs appeasable by calling the DCL files through VisualLISP commands.

\subsection{Development of application functions and macros}

The process behind GUI's widgets is undertaken by application functions or macros. Each function or macro carries a specific task. In this application, functions and macros are used to automatically implement 3D modeling and build a 3D sluice model, instead of step-by-step manual input and operation. As the VisualLISP programming language is able to call AutoCAD commands, system variables and dia$\log$ boxes, the functions written by VisualLISP are able to generate and modify AutoCAD objects as well as to display GUIs to the user in the AutoCAD environment.

To generate a component, there are several steps. First of all, the function for component generation would retrieve input parameters and any other return values from other functions. It would then calculate its own variables. Afterwards, AutoCAD commands would be called to generate (to draw, modify, assemble, etc.) the 3D object based on the function's variables. The object is generated from simple to complex or from $2 \mathrm{D}$ to $3 \mathrm{D}$. 
Each component is constructed through one or several functions, depending on the complexity of the component. Some functions could be re-triggered to carry out a specific task for different components. To avoid repetitiveness of commands in some functions, macro is used to make the program less tedious and less error-prone, as well as to simplify the writing, reading and understanding of the program. Macro is a sequence of commands which are grouped sequentially to act as a single command. Therefore, repetitive commands which carry out the same work could be built into a function and this function could be called each time it is needed.

Let's take a look at the instance in Figure 1-3 and see how to automatically generate a retaining wall using VisualLISP functions. The generation procedure is as follows and depicted in Figure 1-3.

1. Create a function to generate a basic block of the retaining wall

- Call AutoCAD commands to draw the 2D boundary and the extruding path which is used to extrude the boundary to a 3D block.

- Determine the position of the 2D boundary and the extruding path according to an existent mark. Then use the AutoCAD command to place them in the proper positions.

- Call the "extrude" command in AutoCAD to extrude the 2D boundary along the extruding path to complete the block of a retaining wall.

2. Create a function to trim any unnecessary parts of the $3 \mathrm{D}$ block

- From existing data, the blocks which represent unnecessary parts are built following the steps in the first function.

- Call the AutoCAD command to subtract the unnecessary parts from the block.

3. Create a function to completely generate two symmetric walls in accordance with relative components.

- Call the AutoCAD command to mirror the generated wall in the previous function on the opposite side based on the distance between the two symmetric walls. This distance is the width of sluice's slab.
The core data obtained in one function are kept in some global variables in case they need to be used in other functions.

After all components are completely generated, some other functions will be used to assemble all the components together to form a complete 3D sluice model.

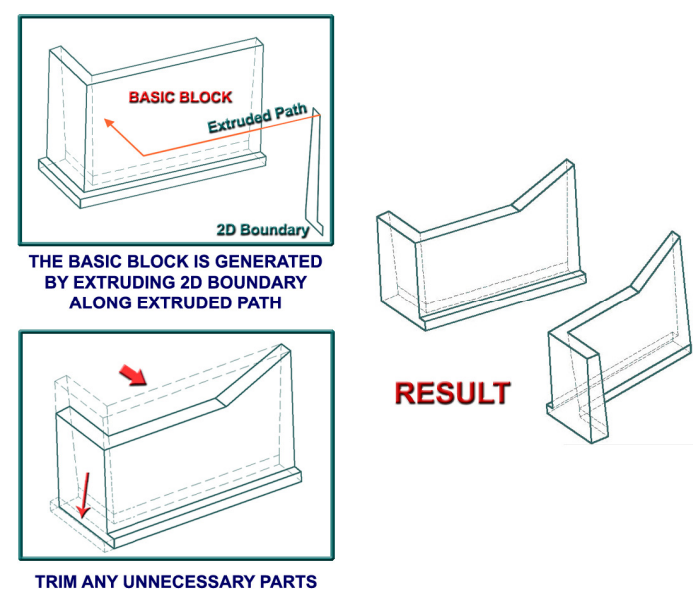

Figure 1-3. The steps of generating retaining walls

\subsection{Test and verification of the application}

Test and verify is a crucial and vital part of the development process. The application has been implemented a number of times with different input data and in different viewports to make sure the application operates correctly with provided data. The result is measured and assessed in each generated model in order to minimize the possibility of errors.

The average generation time for each 3D sluice model is around 8 seconds, which is much shorter than the average required time for step-by-step manual operation by an experienced professional. The average time needed for step-by-step manual operation is about 7 to 8 hours.

\section{RESULTS}

The application result is the generated $3 \mathrm{D}$ sluice model. The 3D sluice model has exactly the same components as the real sluice structure. Therefore, it can be used to render a realistic view of the sluice 
and the model's volume could be used for cost estimation. Figure 1-4 depicts a plain 3D sluice model for volume determination. Some rendered products of application results are introduces in Figure 1-5.

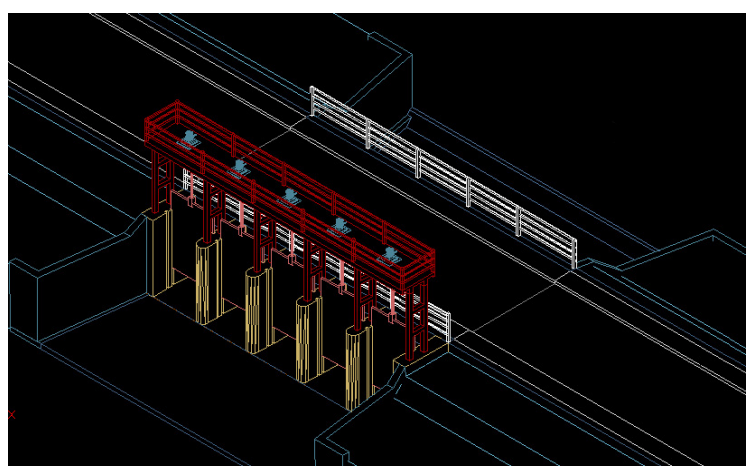

Figure 1-4. A plain 3D sluice model for cost estimation based on the model's volume
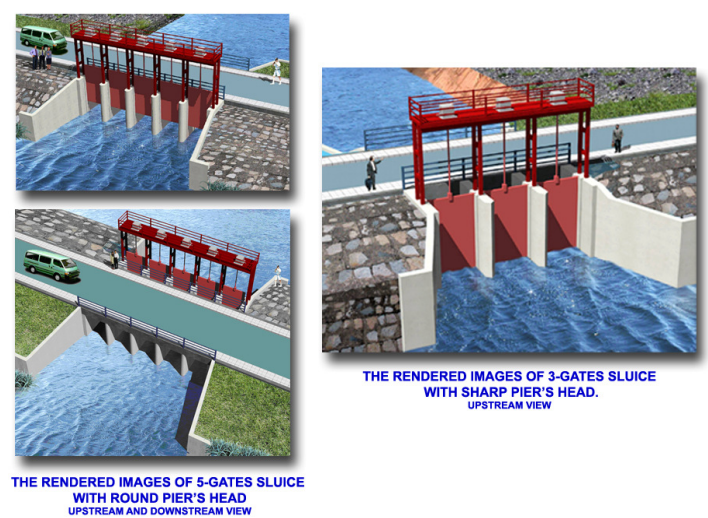

THE RENDERED IMAGES OF 3-GATES SLUICE
WITH SHARP PIER'S HEAD UPSTREAM VEW

Figure 1-5. A rendered product of the 3D sluice model generated by the proposed application

\section{CONCLUSIONS}

In today's highly demanding AEC market and industry, products that could improve productivity, save time, and especially save money are keenly looked for. This paper introduces a way that uses ComputerAided Design (CAD) to automatically generate 3D models for hydraulic construction. As it takes much time, efforts and expert skills to build 3D models for hydraulic facilities, such as dams and sluices, the development of automatic 3D modeling is necessary.

To demonstrate, an application is tailored for $3 \mathrm{D}$ sluice modeling. This application is developed using the VisualLISP programming language and embedded in AutoCAD. Hence, users are able to call the application in the AutoCAD environment as an embedded AutoCAD utility. By using this application, the 3D sluice model will be automatically generated in just a few seconds, saving much time and effort compared to traditional step-by-step manual input and operation.

In fact, there are many 3D modeling software products available in the market [2]. However, those software products are not the tailor-made for any specific area and users need time to get used to the software before engaging in production. This paper also concentrates on this issue, therefore easy-to-use features including user-friendly GUIs and visual indicators are provided in the demonstrated application. As this is a built-in AutoCAD application, its result has the same format as user's work format. Therefore, both types of information are stored in one place and are able to work in one environment. Moreover, users can use AutoCAD utilities to improve the 3D model such as adding additional objects or modifying model's components.

This kind of application is easy to develop as well as to amend. Therefore developers could apply the idea to other areas. For future work, this application could be extended to other hydraulic facilities and augmented with more practical issues such as more interactions with GUIs and data, automatic determination of model's volume and so on.

\section{REFERENCES}

[1.] Autodesk website http://usa.autodesk.com

[2.] Clark A. Cory, Dave Bozell, 3D Modeling for the Architectural Engineering and Construction Industry, GraphiCon Proceedings, 2001 